\title{
A One-Dimensional Theory of Solute Diffusion and Degradation in Elastic Solids
}

\author{
Fernando P. Duda · Angela C. Souza · José M. Barbosa • \\ Leonardo J. Guimarães
}

Received: 13 April 2009 / Published online: 12 May 2009

(C) Springer Science+Business Media B.V. 2009

\begin{abstract}
A theoretical framework for the description of the interaction between diffusion, mechanics, and degradation in elastic solids is developed. To avoid complications that obscure the essential features of these interactions, we work within a one-dimensional setting. A particular specialization of the general theory is selected and a numerical implementation based on the finite-element method, a backward Euler time-stepping scheming, and an operator-splitting algorithm is described. An application involving the time-independent end-loading of a notched cylindrical bar is used to illustrate the ability of the theory to describe some essential features of solute-assisted degradation.
\end{abstract}

Keywords Continuum mechanics · Solute diffusion in solids · Solute-assisted degradation Mathematics Subject Classification (2000) 74A45 - 74F20 - 74F25 - 74R05 - 74R10

\section{Introduction}

In 1878, in his famous memoir "On the Equilibrium of Heterogeneous Substances," Gibbs [1] introduced the idea of a solid that contains fluid components, whereby a fluid

We gratefully thank Eliot Fried for his valuable comments and suggestions.

F.P. Duda $(\bowtie)$

Programa de Engenharia Mecânica, COPPE/UFRJ, Rio de Janeiro, Brazil

e-mail:duda@mecanica.ufrj.br

\section{A.C. Souza}

Departamento de Engenharia Mecânica, PGMEC/UFF, Niterói, Brazil e-mail: angelacris@vm.uff.br

\section{J.M. Barbosa}

Departamento de Engenharia Mecânica, UFPE, Recife, Brazil

e-mail: jmab@demec.ufpe.br

\section{L.J. Guimarães}

Departamento de Engenharia Civil, UFPE, Recife, Brazil

e-mail: leonardo@ufpe.br 
can enter into, move independently through, and distort the solid, which otherwise behaves elastically. As indicated by Li et al. [2], a concrete example of this kind of body is provided by an interstitial solid solution at a sufficiently low temperature, with host and interstitial species playing the role of solid and fluids, respectively. Additional examples can be found in the fields of geology, polymer science, and metallurgy, as Larché \& Cahn [3-5] note.

In this work, we consider solids that contain one fluid component, henceforth referred to as the solute, and extend the idea of Gibbs by including solute-assisted degradation of the solid. This undesirable effect, which may culminate in premature crack initiation and propagation until failure, appears, for instance, in the context of the moisture-induced degradation in polymers (see Weitsman [6]) and the hydrogen-induced degradation in metals (see Glangloff [7]). Specifically, within an one-dimensional, small strain, and isothermal setting, the purpose of this work is twofold:

- to use modern continuum mechanics to develop a framework for the description of the interaction of solute diffusion, deformation, and degradation in elastic solids;

- to specialize the general framework to obtain a simple set of governing equations which demonstrate that the general framework is capable of capturing some of the most essential features of solute-assisted degradation.

Following Fried \& Gurtin [8], we introduce balance laws, a free-energy imbalance that represents the second law of thermodynamics, and constitutive equations. This is carried out as follows. First, we consider as basic the balances of mass for the solid and the solute, and the balances of forces conjugate to the selected independent kinematical fields, namely solid displacement, solid degradation, which is a measure of the degree of degradation, solute flow, and solute density. We then introduce a free-energy imbalance that accounts for energy inflow due to both the power expended by all external forces and the solute supply. The term associated with the solute supply requires the introduction of a chemical potential. We next introduce a thermodynamically consistent constitutive theory based upon the following of assumptions: the solid strain admits an additive decomposition into elastic and solute-induced parts, with the solute-induced strain rate being proportional to the solutedensity rate; the dissipation is due solely to degradation and diffusion; the set of constitutive variables includes elastic strain, degradation, degradation gradient, and solute density. The requirement of thermodynamic consistency implies that the constitutive equations are defined in terms of response functions for the free-energy density, degradation viscosity, friction-like resistance to degradation growth, and solute mobility.

The governing equations of the framework are obtained by merging the aforementioned ingredients. Specifically, the equations for the degradation and the solute flux follow from the corresponding conjugate force balances, whereas the equation for the chemical potential follows from the solute-density-conjugate force balance. These equations can be expressed as follows: (a) the degradation rate is proportional to the positive part of the sum of the negative of the variational derivative of the free-energy density with respect to the degradation, minus the friction-like resistance, plus a contribution due to external agencies, where the coefficient of proportionality is related to the degradation viscosity; (b) the solute flux is proportional to the sum of the chemical potential gradient, where the coefficient of proportionality is related to the solute mobility, plus a contribution due to external agencies; (c) the chemical potential is given by the sum of the derivative of the free-energy density with respect to the solute density, plus a term proportional to the solid stress, plus a term due to external agencies. From the observation that the free-energy density, degradation viscosity, friction-like degradation resistance, and solute mobility can depend on degradation and 
solute density, we reach the conclusion that solute-degradation interactions can be implemented through two distinct routes: a one-way route in which the solute affects the degradation viscosity and/or friction resistance, and the degradation affects the solute mobility; a two-way route in which a solute-degradation interaction term on the free-energy density gives rise simultaneously to solute-assisted degradation and degradation-assisted diffusion. On the other hand, solute-induced strain gives rise to stress-assisted diffusion. This theoretical framework can also accommodate weakly non-local degradation theories through the dependence of the free-energy density on the degradation gradient.

One particular specialization of the general theory is singled out for detailed study. This specialization is based on the following constitutive assumptions: the free-energy density is given as the sum of a degradation-dependent strain energy, a degradation gradient energy, and an entropic contribution due to the mixing of a dilute ideal interstitial solid solution; the degradation viscosity and the solute mobility are constant; frictional resistance is a non-increasing function of the solute content. In this theory, diffusion is driven by the gradients of solute concentration and solid stress. Furthermore, the equation for the degradation is rate-dependent and weakly non-local. We develop a numerical scheme based on the finite-element method, a backward Euler time-stepping scheme, and on a operator-splitting algorithm to solve the weak forms of displacement, degradation, and diffusion equations. An example is given to illustrate the use of the theory for the description of solute-assisted degradation of a cylindrical notched bar.

The present development is similar to that used by Fried \& Sellers [9], where the solute density and solute flow are considered as independent kinematical descriptors. In particular, the use of the solute density as an independent degree of freedom was inspired by Gurtin [10] (see also Podio-Guidugli [11]). The treatment of degradation given here proceeds along the lines given by the authors in Duda \& Souza [12], which is similar to that provided by Costa Mattos \& Sampaio [13] and Frémond \& Nedjar [14] (see also Nedjar [15]), in that degradation is viewed as an independent kinematical process. A preliminary version of this work was presented by the authors in Duda et al. [16]. In a complementary development, Buonsanti, Fosdick and Royer-Carfagni [17] have considered the chemomechanical equilibrium of an elastic bar in contact with a chemically aggressive environment.

The remainder of this paper is organized as follows. The theoretical framework is developed in Sect. 2. A particular specialization of the framework is presented in Sect. 3. The numerical model of this specialization in Sect. 4. An illustrative example is presented in Sect. 5. Finally, some concluding remarks are provided in Sect. 6.

\section{Theoretical Framework}

\subsection{Preliminaries}

Let $\Omega$ be an one-dimensional body identified with the fixed interval $0 \leq x \leq L$. Let $f$ be a smooth function of $x$ and $t$. Choose $f_{x}$ and $\dot{f}$ to denote the partial derivatives of $f$ with respect to $x$ and $t$, respectively.

We suppose that $\Omega$ consists of two components, one of which is conserved and acts as a network through which the other moves independently with velocity $v_{R}$. For definiteness we refer to these components as the solid and solute, respectively. We denote the mass densities, measured per unit length, of the solid and solute by $\rho_{S}$ and $\rho$, respectively. The solid is allowed to undergo deformation and degradation, which are described by the fields $u$ of displacement and $d$ of degradation (or damage). The degradation $d$ ranges between 
zero (pristine material) and unity (completely damaged material), and may be interpreted as representing the fraction of broken atomic bonds. For later reference, we define the strain $\epsilon$ and the composition (or concentration) $c$ through the relations:

$$
\epsilon=u_{x}, \quad c=\frac{\rho}{\rho_{S}} .
$$

The body $\Omega$ can be thought as representing a three-dimensional bar with cross sectional area $A$, in which $\rho_{S}^{*}$ and $\rho^{*}$, the counterparts of $\rho_{S}$ and $\rho$, are given by:

$$
\rho_{S}^{*}=\frac{\rho_{S}}{A}, \quad \rho^{*}=\frac{\rho}{A} .
$$

\subsection{Basic Laws}

Since the solid is conserved, mass balance results in $\dot{\rho}_{S}=0$. Therefore, we assume henceforth that $\rho_{S}$ is given. On the other hand, the mass balance for the solute takes the form of a local field equation:

$$
\dot{\rho}=-J_{x}+h,
$$

where $J:=\rho v_{R}$ is the solute flux, and $h$ represents a given external supply of the solute.

We consider that $u, d, \rho$, and $J$ are independent kinematical descriptors and for this reason introduce four independent force systems. We suppose that these force systems are collectively specified by the contact interactions $\mathbf{t}^{+}$and $\mathbf{t}^{-}$, and by the internal and external body forces $\mathbf{s}^{i}$ and $\mathbf{s}^{e}$. At a point $x \in \Omega, \mathbf{t}^{ \pm}$represents the action of the part $\mathcal{P}^{ \pm}$on the part $\mathcal{P}^{\mp}$, which are obtained by splitting $\Omega$ in two, at the point $x$, i.e., $\mathcal{P}^{+}=(x, L)$ and $\mathcal{P}^{-}=(0, x)$. Further, $\mathbf{t}^{-}(0)$ and $\mathbf{t}^{+}(L)$ represent the contact actions exerted on $\Omega$ by its exterior.

For a given part $\mathcal{D}=\left(x_{1}, x_{2}\right) \subset \Omega$, we define the total force $\mathbf{F}$, and the power expended for all external forces $\mathcal{W}_{e}$ by the relations:

$$
\mathbf{F}(\mathcal{D}):=\mathbf{t}^{-}\left(x_{1}\right)+\mathbf{t}^{+}\left(x_{2}\right)+\int_{\mathcal{D}}\left(\mathbf{s}^{e}(x)-\mathbf{s}^{i}(x)\right) \mathrm{d} \mathbf{x},
$$

and

$$
\mathcal{W}_{e}(\mathcal{D}, \mathbf{v}):=\mathbf{t}^{-}\left(x_{1}\right) \cdot \mathbf{v}\left(x_{1}\right)+\mathbf{t}^{+}\left(x_{2}\right) \cdot \mathbf{v}\left(x_{2}\right)+\int_{\mathcal{D}} \mathbf{s}^{e} \cdot \mathbf{v} \mathrm{dx},
$$

where time-dependence was omitted for simplicity and $\mathbf{v}:=(\dot{u}, \dot{d}, \dot{\rho}, J)$ is the list of generalized velocity field. We remark that $J$ is a rate-type variable. Here, bold-faced letters represent four-tuples of quantities related to the fields $u, d, \rho$, and $J$. The list $\mathbf{f}_{x}$ is formed by the spatial derivatives of the components of $\mathbf{f}$. For two lists $\mathbf{h}$ and $\mathbf{g}, \mathbf{h} \cdot \mathbf{g}$ denotes the sum of the pairwise products of their components.

The aforementioned force systems are supposed to be balanced, i.e.,

$$
\mathbf{F}(\mathcal{D})=\mathbf{0}
$$

for any part $\mathcal{D} \subset \Omega$. This implies that

$$
\mathbf{S}:=\mathbf{t}^{+}=-\mathbf{t}^{-}, \quad \mathbf{S}_{x}-\mathbf{s}^{i}+\mathbf{s}^{e}=\mathbf{0},
$$


with the understanding that above equations hold componentwise. Therefore, defining the four-tuples: $\mathbf{S}=(N, \Pi, \Gamma, \Lambda), \mathbf{s}^{i}=\left(b^{i}, \pi^{i}, \gamma^{i}, \lambda^{i}\right)$, and $\mathbf{s}^{e}=\left(b^{e}, \pi^{e}, \gamma^{e}, \lambda^{e}\right)$, we arrive at the following set of force balances, along with the corresponding field and traction conditions:

- displacement-conjugate force balance

$$
N_{x}-b^{i}+b^{e}=0, \quad N=-N_{0}, \quad N=N_{L},
$$

where $N, b^{e}$ and $b^{i}$ are the displacement-conjugate stress, external body-force, and internal body-force, respectively. $N_{0}$ and $N_{L}$ are the tractions at the boundary of $\Omega$;

- degradation-conjugate force balance

$$
\Pi_{x}-\pi^{i}+\pi^{e}=0, \quad \Pi=-\Pi_{0}, \quad \Pi=\Pi_{L},
$$

where $\Pi, \pi^{e}$ and $\pi^{i}$ are the degradation-conjugate stress, external body-force, and internal body-force, respectively, whereas $\Pi_{0}$ and $\Pi_{L}$ are the tractions at the boundary of $\Omega$;

- solute-density-conjugate force balance

$$
\Gamma_{x}-\gamma^{i}+\gamma^{e}=0, \quad \Gamma=-\Gamma_{0}, \quad \Gamma=\Gamma_{L},
$$

where $\Gamma, \gamma^{e}$ and $\gamma^{i}$ are the solute-density-conjugate stress, external body-force, and internal body-force, respectively, whereas $\Gamma_{0}$ and $\Gamma_{L}$ are the tractions at the boundary of $\Omega$;

- flux-conjugate force balance

$$
\Lambda_{x}-\lambda^{i}+\lambda^{e}=0, \quad \Lambda=-\Lambda_{0}, \quad \Lambda=\Lambda_{L},
$$

where $\Lambda, \lambda^{e}$ and $\lambda^{i}$ are the flux-conjugate stress, external body-force, and internal bodyforce, respectively, whereas $\Lambda_{0}$ and $\Lambda_{L}$ are the tractions at the boundary of $\Omega$.

It also follows from (7) that the external power $\mathcal{W}_{e}$ can be written as:

$$
\mathcal{W}_{e}(\mathcal{D} ; \mathbf{v})=\int_{\mathcal{D}}\left(\mathbf{S} \cdot \mathbf{v}_{x}+\mathbf{s}^{i} \cdot \mathbf{v}\right) \mathrm{dx}=: \mathcal{W}_{i}(\mathcal{D} ; \mathbf{v}),
$$

where $\mathcal{W}_{i}$ is defined as the internal power.

From now on, we assume that the displacement-conjugate internal body-force $b^{i}$ vanishes, which can be justified on the grounds that the internal power is invariant with respect to arbitrary translations.

We also consider as basic a mechanical version of the second law of thermodynamics, namely the free-energy imbalance. It asserts that, for each part $\mathcal{D} \subset \Omega$, its free-energy changes at a rate bounded from above by the power expended by the external forces on the realizable velocity $\mathbf{v}$, plus the energy exchange due to solute supply (see Fried \& Gurtin [8]):

$$
\frac{d}{d t} \int_{\mathcal{D}} \psi d x \leq \mathcal{W}_{e}(\mathcal{D} ; \mathbf{v})+\int_{\mathcal{D}} \mu h \mathrm{dx}
$$

where $\psi$ is the free-energy density and $\mu$ is the chemical potential. Using (12), this version localizes into the dissipation inequality:

$$
\dot{\psi} \leq N \dot{\epsilon}+\Pi \dot{d}_{x}+\pi^{i} \dot{d}+\Gamma \dot{\rho}_{x}+\gamma^{i} \dot{\rho}+\Lambda J_{x}+\lambda^{i} J+\mu h .
$$




\subsection{Constitutive Theory}

The first constitutive assumption adopted is the additive decomposition of the strain $\epsilon$,

$$
\epsilon=\epsilon_{e}+\epsilon_{s}
$$

into its elastic $\epsilon_{e}$ and solute induced $\epsilon_{s}$ parts, where:

$$
\epsilon_{s}=\eta\left(c-c_{0}\right)
$$

with $\eta$ being a positive parameter and $c_{0}$ being a reference concentration. It follows that the dissipation inequality (14) can be written as:

$$
\dot{\psi} \leq N \dot{\epsilon}_{e}+\Pi \dot{d}_{x}+\pi^{i} \dot{d}+\Gamma \dot{\rho}_{x}+\left(\gamma^{i}+\mu+\frac{\eta}{\rho_{S}} N\right) \dot{\rho}+(\Lambda+\mu) J_{x}+\lambda^{i} J .
$$

Guided by the inequality (17), we consider constitutive equations of the form:

$$
\begin{aligned}
& \psi=\hat{\psi}(\mathrm{e}, \mathrm{n}), \quad N=\hat{N}(\mathrm{e}, \mathrm{n}), \quad \Pi=\hat{\Pi}(\mathrm{e}, \mathrm{n}), \quad \pi^{i}=\hat{\pi}^{i}(\mathrm{e}, \mathrm{n}), \quad \Gamma=\hat{\Gamma}(\mathrm{e}, \mathrm{n}), \\
& \gamma^{i}+\mu+\frac{\eta}{\rho_{S}} N=\hat{\gamma}(\mathrm{e}, \mathrm{n}), \quad \Lambda+\mu=\hat{\Lambda}(\mathrm{e}, \mathrm{n}), \quad \lambda^{i}=\hat{\lambda}^{i}(\mathrm{e}, \mathrm{n}),
\end{aligned}
$$

where $(e, n)$ represent the equilibrium and non-equilibrium, or dissipative, constitutive variables. Since we aim for a weakly non-local elastodegradation theory that accounts for compositional effects and diffusion, we assume that:

$$
\mathrm{e}=\left(\epsilon_{e}, d, \rho, d_{x}\right), \quad \mathrm{n}=(J, \dot{d}) .
$$

We assume that degradation occurs under specific circumstances, defined in terms of a loading criterion, to be specified later. To account for this, we also assume that of all response functions presented in (18), $\hat{\pi}^{i}$ is the only one that is not defined at $\dot{d}=0$. In this case, the internal microforce $\pi^{i}$ is not given by a constitutive equation but it is determined by the degradation-conjugate force-balance. For convenience, we assume henceforth that degradation is irreversible, i.e., $\dot{d} \geq 0$.

Following the Coleman-Noll [18] procedure, we require that the constitutive responses must be such that the dissipation inequality (17) holds for all constitutive processes, i.e, for all fields $\left(u, d, \rho, J, \psi, \mu, N, \Pi, \pi^{i}, \Gamma, \gamma^{i}, \Lambda, \lambda^{i}\right)$ satisfying the constitutive assumptions. Thereby, using standard arguments, we conclude that:

1. the constitutive function $\hat{\psi}$ is independent of the non-equilibrium variables $\dot{d}$ and $J$, i.e., $\hat{\psi}(e, n)=\hat{\psi}(e)$;

2. the equilibrium relations

$$
\hat{N}=\frac{\partial \hat{\psi}}{\partial \epsilon_{e}}, \quad \hat{\Pi}=\frac{\partial \hat{\psi}}{\partial d_{x}}, \quad \hat{\Gamma} \equiv 0, \quad \hat{\gamma}=\frac{\partial \hat{\psi}}{\partial \rho}, \quad \hat{\Lambda} \equiv 0
$$

hold;

3. the internal dissipation inequality

$$
\hat{\pi}_{d}(\mathrm{e}, \mathrm{n}) \dot{d}+\hat{\lambda}^{i}(\mathrm{e}, \mathrm{n}) J \geq 0
$$

must hold for all choices of (e, n). 
In (21), $\hat{\pi}_{d}$ is the dissipative part of the internal microforce response, i.e.,

$$
\hat{\pi}_{d}=\hat{\pi}^{i}-\frac{\partial \hat{\psi}}{\partial d} .
$$

Therefore, the constitutive response of a material of the type under consideration is characterized by the functions $\hat{\psi}, \hat{\pi}_{d}$, and $\hat{\lambda}^{i}$. The first of these is independent of $\dot{d}$ and $J$, whereas the second and third must comply with (21).

Now we assume that degradation and diffusion are uncoupled dissipative processes, i.e.,

$$
\hat{\pi}_{d}(\mathrm{e}, \mathrm{n})=\hat{\pi}_{d}(\mathrm{e}, \dot{d}) \quad \text { and } \quad \hat{\lambda}^{i}(\mathrm{e}, \mathrm{n})=\hat{\lambda}^{i}(\mathrm{e}, J)
$$

which implies that each of the terms on the left-hand side of (21) must be non-negative:

$$
\hat{\pi}_{d}(\mathrm{e}, \dot{d}) \dot{d} \geq 0, \quad \hat{\lambda}^{i}(\mathrm{e}, J) J \geq 0 .
$$

Granted sufficient smoothness, and since $\hat{\pi}_{d}$ is not defined at $\dot{d}=0$, it can be shown that the previous inequalities imply the following representations for $\hat{\pi}_{d}$ and $\hat{\lambda}^{i}$ :

$$
\hat{\pi}_{d}(\mathrm{e}, \dot{d})=\hat{\pi}_{1}(\mathrm{e})+\hat{\pi}_{2}(\mathrm{e}, \dot{d}) \dot{d}, \quad \hat{\lambda}^{i}(\mathrm{e}, J)=\hat{\lambda}(\mathrm{e}, J) J
$$

with $\hat{\pi}_{d} \geq 0, \hat{\pi}_{1} \geq 0$, and $\hat{\lambda} \geq 0$. The functions $\hat{\pi}_{1}$ and $\hat{\pi}_{2}$ are called friction-like degradationresistance and degradation-viscosity, respectively, whereas the function $\hat{\lambda}$ is related to the solute mobility. Hereafter, we assume that $\hat{\pi}_{2}>0$ and $\hat{\lambda}>0$.

Now we consider a situation under which degradation occurs. From (9) $1,(20)_{2},(22)$ and $(25)_{1}$, and from the positiveness of $\hat{\pi}_{2}$, it follows that, when $\dot{d}>0$, the inequality

$$
-\delta_{d} \hat{\psi}(\mathrm{e})-\hat{\pi}_{1}(\mathrm{e})+\pi^{e}>0
$$

must hold, where $\delta_{d} \hat{\psi}$ is the variational derivative of the free-energy response with respect to $d$ :

$$
\delta_{d} \hat{\psi}:=\frac{\partial \hat{\psi}}{\partial d}-\frac{\partial}{\partial x}\left(\frac{\partial \hat{\psi}}{\partial d_{x}}\right) .
$$

From now on, we assume that the condition (26) is also sufficient for degradation to occur, thereby representing our choice for the loading condition.

We suppose that degradation manifests itself through a reduction in the capacity of the material to store elastic energy. Therefore, henceforth we assume that the free-energy density contains an interaction term between degradation and elastic strain representing the elastic energy, and that this term is a decreasing function of the degree of degradation. This interaction term describes the reduction in the load bearing capability of the solid produced by degradation. It also dictates how degradation is driven by elastic strain.

\subsection{Governing Equations}

The governing equations for the fields $u, d, \mu, J$, and $\rho$ are obtained through the combination of the aforementioned basic balances and constitutive theory. The corresponding field equations are: 
- the equation for the displacement $u$ is obtained from the force balance $(8)_{1}$, the constitutive equation $(20)_{1}$, and (15), and (16):

$$
N_{x}+b^{e}=0, \quad N=\frac{\partial \hat{\psi}(\mathrm{e})}{\partial \epsilon_{e}}, \quad \epsilon_{e}=u_{x}-\eta\left(c-c_{0}\right) ;
$$

- the equation for the degradation $d$ is obtained from the force balance $(9)_{1}$, the constitutive equations $(20)_{2},(22)$, and $(25)_{1}$, and the condition (26):

$$
\hat{\pi}_{2}(\mathrm{e}, \dot{d}) \dot{d}=\left\langle-\delta_{d} \hat{\psi}(\mathrm{e})-\hat{\pi}_{1}(\mathrm{e})+\pi^{e}\right\rangle,
$$

where \langle\rangle is the McCauley bracket, i.e., $\langle a\rangle=\max (a, 0)$;

- the equation for the chemical potential $\mu$ is obtained from the force balance $(10)_{1}$, and the constitutive equations $(18)_{6}$ and $(20)_{3,4}$ :

$$
\mu=\frac{\partial \hat{\psi}(\mathrm{e})}{\partial \rho}-\frac{\eta}{\rho_{S}} N-\gamma^{e},
$$

with $N$ given by $(20)_{1}$;

- the equation for the solute flux $J$ is obtained from the flux-conjugate force balance (11) and the constitutive equations $(18)_{6},(20)_{3,4}$, and $(25)_{2}$ :

$$
J=\frac{1}{\hat{\lambda}(\mathrm{e}, J)}\left(-\mu_{x}+\lambda^{e}\right)
$$

with $\mu$ given by (30);

- the equation for the solute $\rho$ is obtained from the solute mass balance (3):

$$
\dot{\rho}=-J_{x}+h,
$$

with $J$ given by (31).

The treatment of boundary conditions (at ends $x=0$ and $x=L$ ) is standard. For a field equation that comes from a force balance, boundary conditions involve the prescription of the corresponding kinematical field or traction condition. In particular: the equation for $\mu$ does not involve boundary conditions since, by $(20)_{3}, \Gamma=0$; a boundary condition associated with the equation for $J$ involves the prescription of either $J$ or $\mu$, since, by (18) and $(20)_{3}, \mu=-\Lambda$. On the other hand, a boundary condition for the solute mass equation involves the prescription of either $\rho$ or $J$.

We finalize this section with the following remarks:

- From (29), solute-enhanced degradation can be accounted for by appropriately prescribing $\hat{\pi}_{1}, \hat{\pi}_{2}$, and $\hat{\psi}$. In the latter case, the free-energy density must contain an interaction term between degradation and solute density;

- From (30) it follows that the chemical potential may depend on, among other things, the solute density, elastic strain, and degradation via the free-energy response. In addition, the second term in (30) can be rewritten as $\eta \sigma / \rho_{S}^{*}$, where $\sigma:=N / A$ is the standard stress and $\rho_{S}^{*}$ is the solid density per unit volume;

- It follows that (31) provides a generalization of Fick's law since the solute flux may be driven by the solute density, elastic strain, degradation, and stress gradients. In addition, the solute mobility may depend on elastic strain and degradation; 
- The prescription of $\mu$ implies the prescription of $\rho$, and vice versa. As Larché \& Cahn $([4,5])$ note, when chemical equilibrium prevails at the boundary a constant value for the chemical potential must be prescribed. This does not mean a constant value for $\rho$, since by (30) the chemical potential may involve not only $\rho$ but also stress, elastic strain, and degradation.

\section{Special Theory}

We now present a special theory for the interaction between mechanics, diffusion and degradation. This theory is based on simple choices for the free-energy density and dissipative responses as described below.

We assume that the free-energy $\hat{\psi}$, per unit length of the bar, is given by:

$$
\frac{1}{A} \hat{\psi}(\mathrm{e})=\frac{E}{2}(1-d)^{2} \epsilon_{e}^{2}+w d^{2}+\frac{\kappa}{2} d_{x}^{2}+k_{B} T \rho_{S}^{*} c(\ln c-1),
$$

where $E$ is the Young modulus, $w$ and $\kappa$ are positive parameters, $k_{B}$ is the Boltzmann constant, and $T$ is the temperature. The first and last terms on the right-hand side of (33) represent, respectively, the elastic-energy density and the classical entropic contribution to the free-energy density of a dilute ideal interstitial solid solution. Notice that the elastic energy favors the local state $d=1$, the second term on the right-hand side favors the local state $d=0$, and the third term penalizes rapid spatial variation and smears out the transition zone in which $d$ varies between 0 and 1 . Aside from the effect of the solute, this choice for the free-energy density appears in the context of the variational approximation of brittle fracture given by Ambrosio \& Tortorelli [19] (see also Braides [20]).

As for the dissipative responses, we assume that:

$$
\hat{\pi}_{1}=2(1-d) \hat{w}_{c}(c) A, \quad \hat{\pi}_{2}=\beta A, \quad \hat{\lambda}=\frac{1}{M \rho},
$$

where $\hat{w}$ is a non-increasing function of $c$, and $\beta$ and $M$ are positive parameters determining the kinetics of degradation and the mobility of the solute, respectively.

For the sake of simplicity, we do not consider the fields $\pi^{e}, \lambda^{e}, \gamma^{e}$, and $h$. With the aforementioned assumptions, (28)-(32) become:

$$
\left\{\begin{array}{l}
N_{x}+b^{e}=0, \\
\beta A \dot{d}=\left\langle\Pi_{x}-\pi\right\rangle, \\
\rho_{S}^{*} A \dot{c}=-J_{x},
\end{array}\right.
$$

where:

$$
\begin{aligned}
\sigma & =\frac{N}{A}=E(1-d)^{2} \epsilon_{e}, \quad \epsilon_{e}=u_{x}-\eta\left(c-c_{r}\right), \\
\pi & =2 w A d-A(1-d)\left(E \epsilon_{e}^{2}-2 \hat{w}_{c}\right), \quad \Pi=\kappa A d_{x}, \\
J & =-\frac{D \rho_{S}^{*}}{k_{B} T} A c \mu_{x}, \quad \mu=k_{B} T \ln c-\frac{\eta}{\rho_{S}^{*}} \sigma, \quad D=M k_{B} T,
\end{aligned}
$$

where $D$ is the diffusion coefficient. Notice that, from $(36)_{1,3}$, solute diffusion is driven by the stress gradients, which implies that, since $\eta>0$, stress-assisted diffusion favors solute migration from lower to higher stressed locations. 
The set of equations (35) provide the governing equations for the fields $u, d$, and $c$, which must be supplemented by initial conditions on $d$ and $c$, and, at each boundary point, prescriptions for $u$ or $N, d$ or $\Pi$, and $c$ or $J$.

\section{Numerical Model}

Now we briefly describe the steps involved in the construction of the numerical model, which is based on the finite-element method, a backward Euler time-stepping scheme, and an operator-splitting algorithm.

The governing equations written in their weak forms are:

$$
\begin{aligned}
& \int_{0}^{L}\left(N v_{x}-b^{e} v\right) \mathrm{dx}+N_{0} v(0)-N_{L} v(L)=0, \\
& \int_{0}^{L}\left(J \varphi_{x}-A \rho_{S}^{*} \dot{c} \varphi\right) \mathrm{dx}+J_{0} \varphi(0)-J_{L} \varphi(L)=0, \\
& \int_{0}^{L}\left(\Pi \alpha_{x}+(\beta A \dot{d}+\pi) \alpha\right) \mathrm{d} \mathrm{x}+\Pi_{0} \alpha(0)-\Pi_{L} \alpha(L)=0, \quad \dot{d} \geq 0,
\end{aligned}
$$

where $v, \varphi$, and $\alpha$ represent arbitrary test functions and $N, \Pi, \pi$, and $J$ are as given in (36). The test functions are assumed to belong to appropriate function spaces and to vanish at a boundary point where essential boundary conditions are prescribed. As in Nedjar [15], the condition $\dot{d} \geq 0$ is imposed at the outset in $(37)_{3}$.

Approximations for the fields $u, c, d, v, \varphi$, and $\alpha$ are obtained via the finite-element method using the same underlying mesh. After inserting these approximations into (37), we arrive at the following semi-discrete systems of equations:

$$
\begin{aligned}
& \mathbf{F}_{u}(\mathbf{u}, \mathbf{c}, \mathbf{d})=\mathbf{0}, \\
& \mathbf{F}_{c}(\mathbf{u}, \mathbf{c}, \dot{\mathbf{c}}, \mathbf{d})=\mathbf{0}, \\
& \mathbf{F}_{d}(\mathbf{u}, \mathbf{c}, \mathbf{d}, \dot{\mathbf{d}})=\mathbf{0},
\end{aligned}
$$

where $\mathbf{u}, \mathbf{c}$, and $\mathbf{d}$ are vectors containing the unknown nodal values for $u, c$, and $d$. The functions $\mathbf{F}_{u}, \mathbf{F}_{c}$, and $\mathbf{F}_{d}$, viewed as vector valued, have the same dimensions of $\mathbf{u}, \mathbf{c}$, and $\mathbf{d}$, respectively. The temporal discretization of this set of equations is carried out by using the implicit Euler scheme. This results in the following set of equations for each time instant $t_{k}$ :

$$
\begin{aligned}
& \mathbf{F}_{u}\left(\mathbf{u}^{k}, \mathbf{c}^{k}, \mathbf{d}^{k}\right)=\mathbf{0}, \\
& \mathbf{F}_{c}\left(\mathbf{u}^{k}, \mathbf{c}^{k}, \dot{\mathbf{c}}^{k}, \mathbf{d}^{k}\right)=\mathbf{0}, \\
& \mathbf{F}_{d}\left(\mathbf{u}^{k}, \mathbf{c}^{k}, \mathbf{d}^{k}, \dot{\mathbf{d}}^{k}\right)=\mathbf{0},
\end{aligned}
$$

where, for a time dependent function $f$, we consider the following approximation:

$$
\dot{f}^{k}=\frac{f^{k}-f^{k-1}}{t_{k}-t_{k-1}}, \quad \text { where } f^{k}=f\left(t_{k}\right), \dot{f}^{k}=\dot{f}\left(t_{k}\right) .
$$

The computational problem is to determine $\mathbf{u}^{k}, \mathbf{c}^{k}$, and $\mathbf{d}^{k}$ given $\mathbf{c}^{k-1}$ and $\mathbf{d}^{k-1}$. This is solved by using a staggered scheme comprising the following steps: 
(i) assume $\mathbf{c}^{k-1}$ and $\mathbf{d}^{k-1}$ as trial solutions for $\mathbf{c}^{k}$ and $\mathbf{d}^{k}$;

(ii) obtain the trial solution for $\mathbf{u}^{k}$ by solving (40) $)_{1}$;

(iii) update the trial solution for $\mathbf{c}^{k}$ by solving $(40)_{2}$;

(iv) update the trial solution for $\mathbf{d}^{k}$ by solving (40) 3 ;

(v) if convergence is attained, update $\mathbf{u}^{k}, \mathbf{c}^{k}$, and $\mathbf{d}^{k}$ using their trials, otherwise, go to Step (ii) repeating the subsequent steps.

\section{Example}

Tensile notched specimens play a prominent role in experimental investigations of hydrogenassisted cracking of metals. This is a major concern in regard to high-strength alloys applications (see Glangloff [7]). A notched specimen under tensile loading undergoes a nonuniform stress distribution that promotes hydrogen diffusion towards the notch. This may result in premature crack initiation and propagation until failure if a high enough level of hydrogen accumulation is achieved. These considerations motivate the following illustrative example.

We consider the problem of solving (35)-(36) for $u, d$, and $c$ with the following initial and boundary conditions: $d(x, 0)=0$ and $c(x, 0)=c_{0}$, with $c_{0}$ constant; $J(0, t)=$ $J(L, t)=0, u(0, t)=0$, and $N(L, t)=P$. The initial conditions mean that at the outset, the bar is pristine and contains a uniform solute concentration, whereas the boundary conditions mean that the bar is impermeable, and has one end fixed and the other end subjected to a fixed load $P$. We also take $b^{e}=0$ and $\Pi(0, t)=\Pi(L, t)=0$, which, by $(36)_{2}$, are equivalent to take homogeneous Neumann boundary conditions for $d$. The bar has a circular cross section with radius given by:

$$
r= \begin{cases}r_{0}-\sqrt{r_{n}^{2}-\left(\frac{L}{2}-x\right)^{2}}, & \left|x-\frac{L}{2}\right|<r_{n}, \\ r_{0}, & \text { otherwise }\end{cases}
$$

as a result of presence of a circular notch of radius $r_{n}$ located on its central portion, where $r_{0}$ is constant.

We now discuss some general features of the problem just defined. Initially, we observe that, since $b^{e}=0$, we can replace (35) $)_{1}$ by the statement that $N=\sigma A$ is spatially constant. Then, from the boundary condition $N(L, t)=P$, it follows that:

$$
\sigma(x, t)=\frac{P}{A(x)},
$$

which shows that $\sigma$ is time-independent and given in terms of the applied load $P$ and crosssectional area $A$. In addition, the impermeability condition implies that:

$$
\int_{0}^{L} c d x=c_{0} L
$$

which means that the solute content is conserved. Notice also that the governing equation for $c$, which is obtained by combining $(35)_{3},(36)_{3}$, and $(36)_{4}$, can be written as:

$$
A \dot{c}=D\left(A\left(c_{x}-\gamma c \sigma_{x}\right)\right)_{x}
$$


where $\gamma=\eta / \rho_{S}^{*} k_{B} T$. This implies that, for the problem under consideration, the field $c$ can be obtained independently from $u$ and $d$. In particular, the steady-state solution $c_{s}$ of (44) is given by:

$$
c_{s}=K e^{\gamma \sigma},
$$

where, by (43),

$$
K=c_{0} L\left(\int_{0}^{L} e^{\gamma \sigma} d x\right)^{-1} .
$$

We seek to establish a condition under which degradation occurs. This condition should involve the applied load $P$, the initial concentration $c_{0}$, and various geometrical and constitutive properties of the bar. In addition, we present a numerical example describing the solute-assisted degradation of the bar up to the instant of fracture.

\subsection{A Condition for Degradation}

We first consider the existence of particular solutions of (35)-(36) for which $d$ is constant and equal to zero. In this case, it follows that $(35)_{2}$ reduces to the inequality:

$$
\frac{E}{2} \epsilon_{e}^{2} \leq \hat{w}_{c}(c)
$$

From this inequality we conclude that $d$ remains equal to zero provided that the elastic energy density $E \epsilon_{e}^{2} / 2$ is below a critical threshold defined by $\hat{w}_{c}$. Using (36) $)_{1}$, we can rewrite (47) as:

$$
\sigma \leq \sqrt{2 E \hat{w}_{c}(c)}:=\hat{\sigma}_{c}(c),
$$

which shows that $d$ remains equal to zero provided that the stress $\sigma$ is below a critical threshold defined by the critical stress $\hat{\sigma}_{c}(c)$.

Now, we evaluate the inequality (48) at $x=L / 2$ and use (42) to yield:

$$
P \leq \hat{\sigma}_{c}\left(c^{*}(t)\right) \pi\left(r_{0}-r_{n}\right)^{2},
$$

where $c^{*}(t):=c(L / 2, t)$. Since $\hat{w}_{c}$ is a non-increasing function of $c$ and $c^{*}$ attains its maximum for a steady-state solution of (44), it follows from (49) that:

$$
P \leq \hat{\sigma}_{c}\left(c_{\max }\right) \pi\left(r_{0}-r_{n}\right)^{2}
$$

where $c_{\max }=c_{s}(L / 2)$ and $c_{s}$ is a steady-state solution of (44) given by (45).

We may therefore conclude that if $d=0$ identically satisfies (35)-(36), it follows that the inequality (50) holds. The opposite statement gives a necessary condition under which degradation occurs. Therefore, degradation occurs if

$$
P>\hat{\sigma}_{c}\left(c_{\max }\right) \pi\left(r_{0}-r_{n}\right)^{2},
$$

where $c_{\max }=c_{s}(L / 2)$ and $c_{s}$ given by (45). 
Table 1 Parameters

\begin{tabular}{ll}
\hline$L$ & $0.1 \mathrm{~m}$ \\
$r_{0} / L$ & $9.0 \times 10^{-2}$ \\
$r_{n} / L$ & $2.5 \times 10^{-2}$ \\
$E$ & $200.0 \mathrm{GPa}$ \\
$\rho_{S}^{*}$ & $1.28 \times 10^{29} \mathrm{Fe} / \mathrm{m}^{3}$ \\
$\eta$ & $0.0937 \mathrm{Fe} / \mathrm{H}$ \\
$D$ & $1.0 \times 10^{-8} \mathrm{~m}^{2} / \mathrm{s}$ \\
$c_{0}$ & $5.5 \times 10^{-6} \mathrm{H} / \mathrm{Fe}$ \\
$k_{B} T$ & $4.14 \times 10^{-21} \mathrm{~J} / \mathrm{H}$ \\
$\bar{c}$ & $8.31 \times 10^{-6} \mathrm{H} / \mathrm{Fe}$ \\
$\hat{w}_{c}(0)$ & $10 \mathrm{MPa}$ \\
$\bar{w}_{c}(\bar{c})$ & $1.6 \mathrm{MPa}$ \\
$\beta / \hat{w}_{c}(0)$ & $\left\{10^{2}, 10^{3}\right\} \mathrm{s}$ \\
$w / \hat{w}_{c}(0)$ & $\{0,1\}$ \\
\hline
\end{tabular}

\subsection{Numerical Solution}

We now employ the numerical formulation presented before to obtain an approximate solution for (35)-(36), with the boundary and initial conditions presented at the beginning of this section. The solution describes the entire degradation process undergone by the bar until it fractures in the middle.

The parameters adopted in the simulations are given in Table 1. The mechanochemical parameters are typical for $\mathrm{Fe}-\mathrm{H}$ system. The value of $c_{0}$ corresponds to a electrochemical charging of hydrogen during thirty five hours in a $0.1 \mathrm{~N} \mathrm{NaOH}$ solution at a current density of 0.3 Ampere $/ \mathrm{m}^{2}$, as described in Wang et al. [22]. The remaining mechanochemical parameters can be found in Sofronis [21]. As for the constitutive quantities related to degradation, we consider that $\hat{w}_{c}$ decreases linearly in the interval $[0, \bar{c}]$ and remains equals to $\hat{w}(\bar{c})$ otherwise. The selected values for $\bar{c}, \hat{w}_{c}(0)$, and $w_{c}(\bar{c})$ correspond to a decreasing of the tensile fracture stress of a specimen from $2000 \mathrm{MPa}$ when uncharged to $800 \mathrm{MPa}$ when charged with a hydrogen content of $0.15 \mathrm{ppm}$. Aside from the assumed shape of $\hat{w}_{c}$, these data resemble the experimental data obtained by Wang et al. [22]. The value for $\kappa$ corresponds to the choice of the length scale $\sqrt{\kappa / w_{c}(0)} \approx 0.3 \mathrm{~mm}$. The values of $\beta$ and $w$ were chosen for illustrative purposes. The applied load is chosen to be of the form

$$
\frac{P}{\pi\left(r_{0}-r_{n}\right)^{2}}=0.95 \hat{\sigma}_{c}\left(c_{0}\right) \text {. }
$$

The results are described in what follows.

First, we use (42) to obtain the stress distribution depicted in Fig. 1. If degradation were not considered, the stress concentration would trigger solute migration for the highly stressed region, which in this case occurs in a neighborhood of the notch centered at the midpoint $x=L / 2$ along the axis of the bar, until achievement of the steady-state, as indicate in Fig. 2. The steady-state solution $c_{s}$ corresponds to the instant $t_{c}^{\infty}$.

When degradation is allowed, the solute accumulates until the time $t=t_{F}$, which is defined by the condition $d\left(L / 2, t_{F}\right)=1$ and is interpreted as time to failure. However, initially the solute accumulates without any degradation until $t=t_{I}$ in which:

$$
\frac{P}{\pi\left(r_{0}-r_{n}\right)^{2}}=\hat{\sigma}_{c}\left(c^{*}\left(t_{I}\right)\right) .
$$


Fig. 1 Stress distribution normalized by $\hat{\sigma}_{c}\left(c_{0}\right)$ obtained through (42)

Fig. 2 Solute distribution at the instants $t_{c}^{1}<t_{c}^{2}<t_{c}^{\infty}$, where $t_{c}^{\infty}$ corresponds to the steady-state
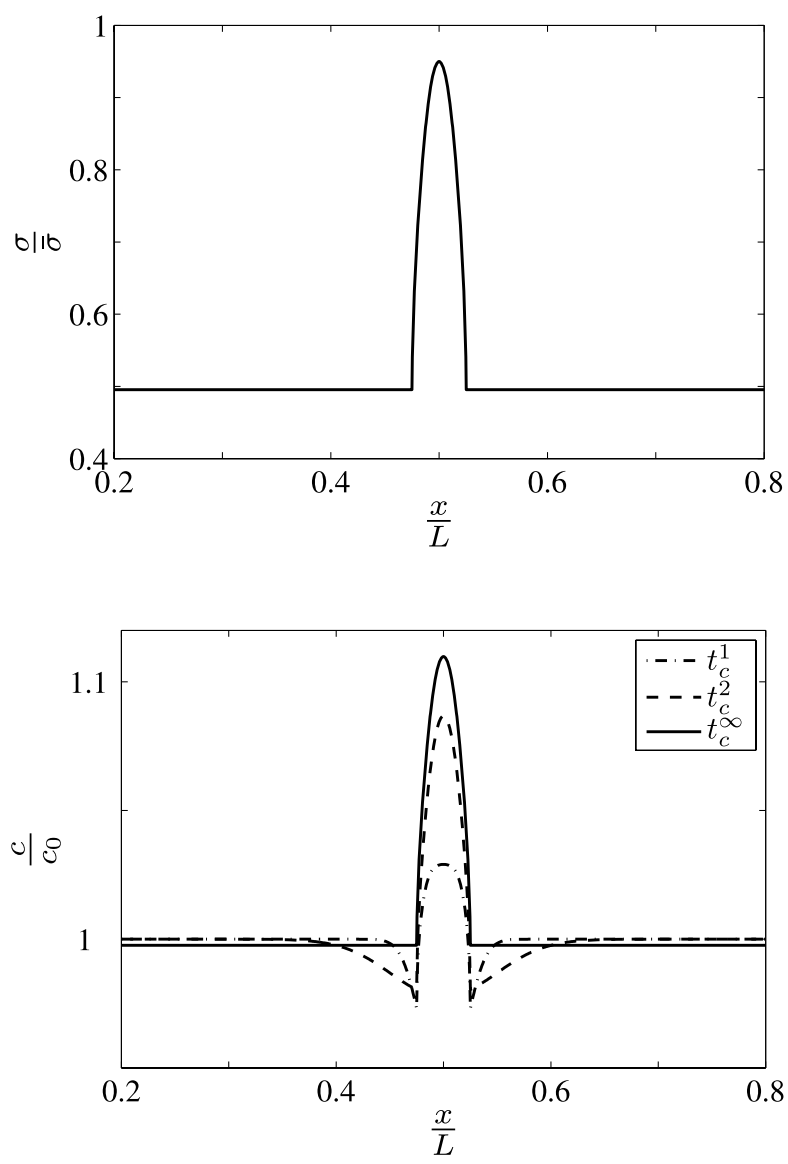

Fig. 3 Evolution of solute concentration and degradation at $x=\frac{L}{2}$. The solid lines represent the degradation evolution for two distinct values of $\beta$. The dashed line represents the evolution of $(c-1)$ normalized by its maximum value

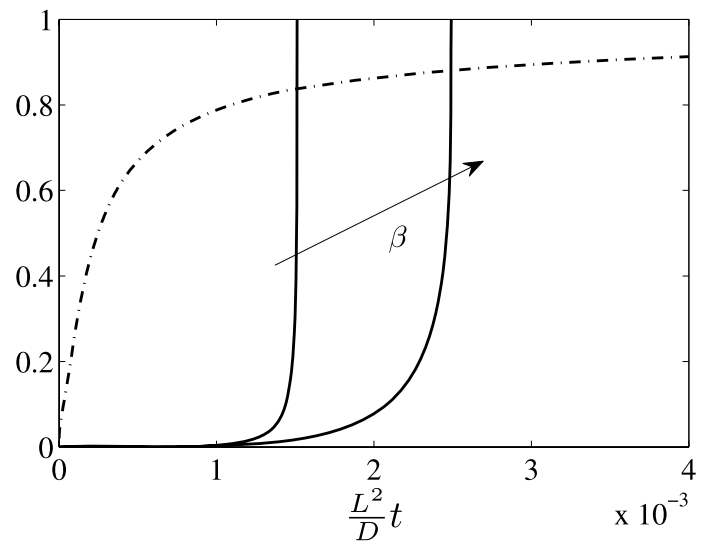

Subsequently to $t_{I}$, degradation commences and propagates until failure occurs. These facts are illustrated in Figs. 3 and 4, in which the solid lines represent the evolution of the degradation field at $x=L / 2$, whereas the dashed line represents the evolution of $(c-1)$ normalized 
Fig. 4 Evolution of solute concentration and degradation at $x=\frac{L}{2}$. The solid lines represent the degradation evolution for two distinct values of $w$. The dashed line represents the evolution of $(c-1)$ normalized by its maximum value

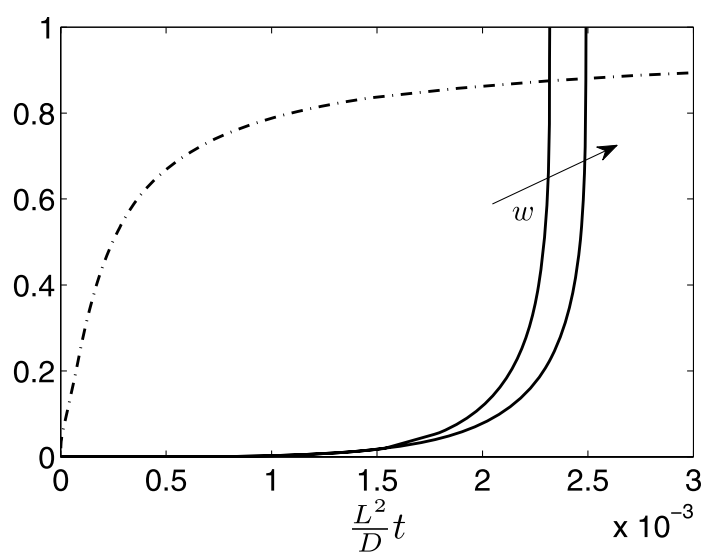

Fig. 5 Degradation distribution at the instants $t_{d}^{1}<t_{d}^{2}<t_{F}$, where $t_{F}$ is the instant at which failure occurs

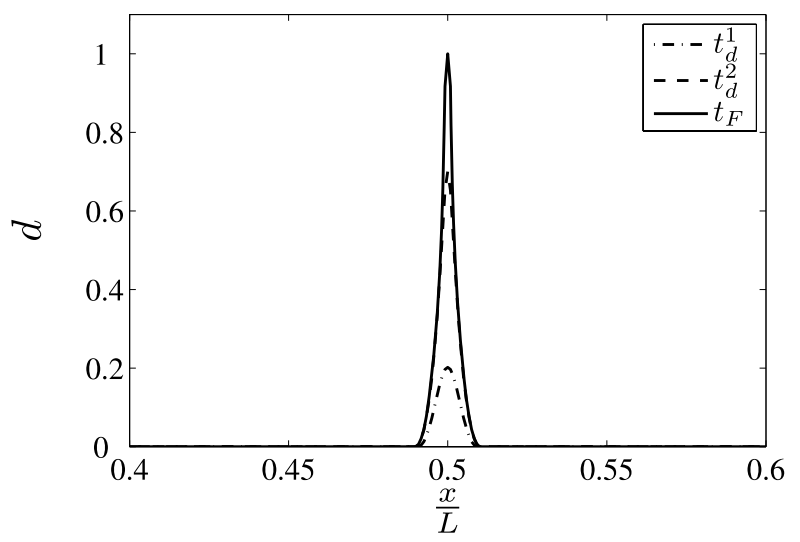

by its maximum value, also evaluated at $x=L / 2$. Figures 3 and 4 also indicate that the time to failure $t_{F}$ increases with both $\beta$ and $w$. Figure 5 depicts the degradation distribution at the instants: $t_{d}^{1}<t_{d}^{2}<t_{F}$, where $t_{F}$ is the instant at which failure occurs. Notice that degradation is confined to a small central region.

\section{Conclusions}

In this paper we presented a one-dimensional theoretical framework for the description of the interaction between solute diffusion and degradation in elastic solids. The solid-solute system was kinematically described by the following independent fields: solid displacement, solid degradation, solute density, and solute flow. Then four force balances, one for each independent kinematical descriptor, which along with the mass balances for the solid and solute, form the set of balance equations of the theory. A thermodynamically compatible set of constitutive equations was developed, the main assumptions of which are: strain decomposes additively into elastic and solute-induced parts; degradation and diffusion are the only source of dissipation. A simplified theory was selected and illustrated through an analysis carried out for a notched bar. In particular, a numerical example showed how interaction between mechanics, diffusion, and degradation work together leading to the breakage 
of the bar. The numerical model was based on the finite-element method, a backward Euler time-stepping scheme, and an operator-splitting algorithm.

Acknowledgements The first author gratefully acknowledges the support by CNPq (485866/2006-4 and 484579/2006-1) and FAPERJ (E-26/171.599/2004). The second author gratefully acknowledges the support by the FAPERJ (E-26/170.292/2006). The final version of this work was written while the first two authors were Visiting Scholars at the Mechanical Engineering Department at McGill University, whose hospitality and support the authors wish to acknowledge. The first two authors also acknowledge CNPq (201080/2008-7) and CAPES (0720-08-9) for post-doctoral fellowships.

\section{References}

1. Gibbs, J.W.: The Scientific Papers of J. Williard Gibbs, vol. 1, Thermodynamics. Ox Bow Press, Woodbridge (1993)

2. Li, J.C.M., Oriani, R.A., Darken, L.S.: The thermodynamics of stressed solids. Z. Phys. Chem. Neue Folg 49, 271-290 (1966)

3. Larché, F.C., Cahn, J.W.: A linear theory of thermochemical equilibrium of solids under stress. Acta Metall. 21, 1051-1063 (1973)

4. Larché, F.C., Cahn, J.W.: The effect of self-stress on diffusion in solids. Acta Metall. 30, 1835-1845 (1982)

5. Larché, F.C., Cahn, J.W.: The interactions of composition and stress in crystalline solids. Acta Metall. 33, 331-357 (1985)

6. Weitsman, Y.J.: Effects of Fluids on Polymeric Composites-A Review. University of Tennessee Report MAES98-5.0-CM (August 1998)

7. Gangloff, R.P.: Hydrogen assisted cracking of high strength alloys. In: Comprehensive Structural Integrity, vol. 6, pp. 31-101. Elsevier, New York (2003)

8. Fried, E., Gurtin, M.: Coherent solid-state phase transitions with atomic diffusion: A thermomechanical treatment. J. Stat. Phys. 95(5-6), 1361-1427 (1999)

9. Fried, E., Sellers, S.: Theory for atomic diffusion on fixed and deformable crystal lattices. J. Elast. 59, $67-81(2000)$

10. Gurtin, M.E.: Generalized Ginzburg-Landau and Cahn-Hilliard equations based on a microforce balance. Physica D 92, 178-192 (1996)

11. Podio-Guidugli, P.: Models of phase segregation and diffusion of atomic species on a lattice. Ric. Mat. $\mathbf{5 5}, 105-118(2006)$

12. Duda, F.P., Souza, A.C.: On a continuum theory of brittle materials with microstructure. Comput. Appl. Math. 23, 327-343 (2004)

13. Costa Mattos, H., Sampaio, R.: Analysis of the fracture of brittle elastic materials using a continuum damage model. Struct. Eng. Mech. 13(5), 411-427 (1995)

14. Frémond, M., Nedjar, B.: Damage, gradient of damage and the principle of virtual power. Int. J. Solids Struct. 33, 1083-1103 (1996)

15. Nedjar, B.: Elastoplastic-damage modelling including the gradient of damage: formulation and computational aspects. Int. J. Solids Struct. 38, 5421-5451 (2001)

16. Duda, F.P., Souza, A.C., Guimarães, L.J., Barbosa, J.M.: An one dimensional coupled model for deformation, degradation and solute diffusion in elastic solids. In: Alves, M., da Cost Mattos, H.S. (eds.) Mechanics of Solids in Brazil. Brazilian Society of Mechanical Sciences and Engineering, Rio de Janeiro (2007)

17. Buonsanti, M., Fosdick, R., Royer-Carfagni, G.: Chemomechanical equilibrium of bars. J. Elast. 84, $167-188$ (2006)

18. Coleman, B.D., Noll, W.: The thermodynamics of elastic materials with heat conduction and viscosity. Arch. Ration. Mech. Anal. 13(1), 167-178 (1963)

19. Ambrosio, L., Tortorelli, V.M.: On the approximation of free discontinuity problems. Boll. Un. Mat. Ital. B 7(6), 105-123 (1992)

20. Braides, A.: $\Gamma$-convergence for Beginnners. Oxford University Press, Oxford (2005)

21. Sofronis, P.: The influence of mobility of dissolved hydrogen on the elastic response of a metal. J. Mech. Phys. Solids 43, 1385-1407 (1995)

22. Wang, M., Akiyama, E., Tsuzaki, K.: Effect of hydrogen on the fracture behavior of high strength steel during slow strain rate test. Corros. Sci. 49, 4081-4097 (2007) 